\title{
Mathematical Model for Elliptic Torus of Automotive Torque Converter and Fundamental Analysis of Its Effect on Performance
}

\author{
Chunbao Liu, Changsuo Liu, and Wenxing Ma \\ School of Mechanical Science and Engineering, Jilin University, Changchun 13002, China \\ Correspondence should be addressed to Wenxing Ma; mawx@jlu.edu.cn
}

Received 15 January 2015; Accepted 21 April 2015

Academic Editor: Kacem Chehdi

Copyright (C) 2015 Chunbao Liu et al. This is an open access article distributed under the Creative Commons Attribution License, which permits unrestricted use, distribution, and reproduction in any medium, provided the original work is properly cited.

\begin{abstract}
Passenger car torque converters have been designed with an increasingly flatter profile in recent years for the purpose of achieving a weight saving and more compact size. However, a flatter design tends to result in the reduced hydrodynamic performance. To improve its performance, a new flat torus of elliptic type method concentrating on the solution to flat TC was put forward, and four torque converters of different flatness ratios were designed to judge the superiority of the flat torus design method. The internal flow characteristics were numerically investigated using CFD codes and resulted in good agreement with experimental data. The results indicate that the main cause of this performance degradation can be attributed to deterioration of the velocity fields of the pump, and a case of flatness ratio 0.8 illustrates the reason that the performance designed by the flat torus design method based on the elliptic shape is more excellent than that of the traditional one. Furthermore, this study proposed a structure of removal of inner ring to improve the performance of torque converter.
\end{abstract}

\section{Introduction}

Internal combustion engine vehicles that employ automatic transmissions typically include a torque converter (TC) positioned between the engine and the transmission of the vehicle. A torque converter is a fluid coupling device typically including an impeller at the input coupled to an output shaft of the engine and a turbine at the output coupled to the input shaft of the transmission. The smooth transmission of torque is provided and torque amplification at the low speeds is accomplished with the device. A pump, a turbine, and a stator are all parts of a torque converter. The working fluid in the closed system is light oil and the pump, which in many ways acts as a centrifugal pump energizing the fluid, while the turbine extracts the energy from the fluid.

Most passenger cars with small and medium displacement engines have adopted a front-wheel-drive layout in recent years, and the gears of gearbox and output torque of passenger cars equipped with AT are continuously increasing. These components, together with locking clutch and torsional vibration damper, need more space $[1,2]$. TCs accordingly have been designed with an increasingly narrower profile for the purpose of achieving a smaller axial size, which also translates into weight saving. The TC structure is bound to be flat, and the performance needs constant improvement, such as Exedy flat torque converters [3], whose maximum width of circle is shortened to $30 \%$ but whose torque is increased to $106 \%$.

Several numerical and experiment attempts have been made to elucidate the flow characteristics inside TC passages. Ejiri and Kubo [4] investigated the hydraulic performance of a torque converter using a five-hole Pitot tube. Results indicated that the pump is a major source of loss at the most frequently utilized speed ratio range. Flow visualization and a three-dimensional flow analysis were used to investigate the cause of the losses in the pump. Marathe and Lakshminarayana [5] also obtained average and unsteady pressure and velocity data upstream and downstream of a torque converter pump using the high frequency response five-hole probe. Separation zones were found near the core at the 0.800 speed ratio and at the shell at the 0.065 speed ratio. The complexity of the flow in TC has led to the increasing 


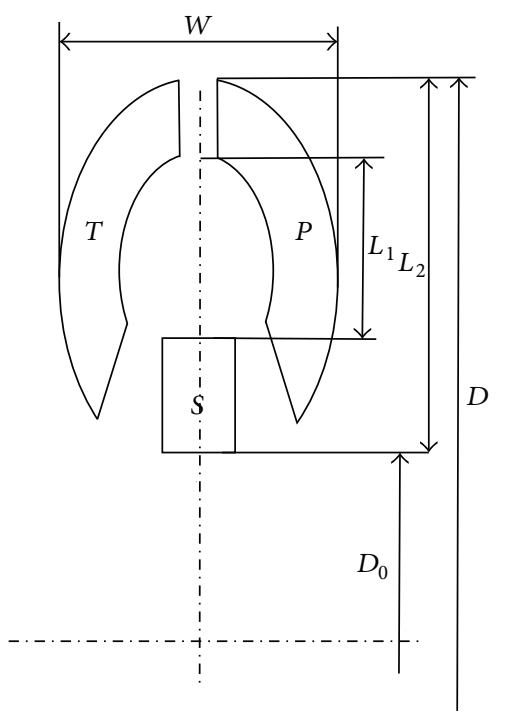

(a) Torus

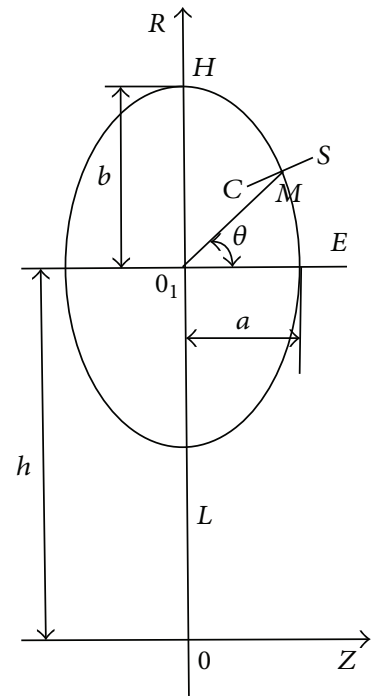

(b) Intermediate flow line

FIGURE 1: Dimensions in torus cross section.

reliance on computational fluid dynamics (CFD) as a design tool for improving efficiency. A three-dimensional, transient, incompressible, viscous Navier-Stokes code was employed by Dong et al. [6] to predict the performance of TC and numerical solution was found to be consistently within 5\% of the experimental data. Kim et al. [7] made a performance estimation model for a TC using correlation between the internal flow field and energy loss coefficient. Jung et al. [8] performed a comparative study to investigate the effects of different methods for handling the relative motion of parts and improved the accuracy.

The analysis of flow characteristic of a TC is quite complex due to several conditions. First, the flow field changes largely over the typical operating range; for example, incidence flow angles to all of the component blades change drastically [9]. Second, the flow is also turned to the transverse direction in a short distance in both the pump and turbine; namely, flow enters these components in the axial direction and turned to the radial direction and, then, rapidly turned into the reverse axial direction, leading to further separation [10-13]. The TC pump is, therefore, a complex mixed-flow variety of hydraulic turbomachine with a variety of forces acting on the fluid particles $[14,15]$.

Only a few analytical studies have focused on the influence of flatness ratio of TC on hydrodynamic performance; Ejiri and Kubo [16] manufactured and tested four TCs of different flatness ratios to evaluate the change in their overall performance. However, the design method they adopted is still a traditional torus design method which is suitable to various types of TCs; that is, the shell is firstly determined according to the flow section area and then the average point method is adopted to achieve the inner and middle streamlines. However, the inherent defects exist, such as the poor stability of the flow area along the axial flow line, the inconsistent change in curvature, and the roughness of the flow line. Furthermore, the traditional defined flatness ratio always loses the details of characteristics on the whole circle.

In the present work, a new design method of elliptic type has addressed the above shortcomings; the performance characteristics of TC of flatness ratio 0.8 were numerically investigated through the CFD approaches. It has demonstrated the superiority of new method and also illustrated the internal flow mechanism of performance improvement.

This study is organized as follows. In Section 2, a new design method of elliptic type concentrating on the solution to flat TC is proposed. Then, the performance characteristics of TC are investigated using CFD codes and experiment in Section 3, respectively. The overall performance of different flatness ratio and the superiority of the new method are analyzed and discussed in Section 4. Furthermore, Section 5 presents a structure in which inner ring is removed. Finally, the specific conclusions of this paper are given in Section 6.

\section{New Design Method of Flat Torque Converter Torus}

2.1. Parameters of the Intermediate Line Parameter. In the new method, the intermediate flow line in circle is defined as the ellipse type in the ROZ coordinate system; the torus of TC and intermediate flow line are shown in Figure 1; the ratio of the short axis $a$ to the long axis $b$ is defined as the flatness ratio, namely, FR $=a / b$, while the flatness ratio was previously defined as the ratio of maximum width $W$ and maximum effective circle diameter $D$.

The elliptic equation of the intermediate flow line is defined as

$$
\frac{Z^{2}}{a^{2}}+\frac{(R-h)^{2}}{b^{2}}=1
$$


where $Z=a \cos \theta$ and $R=b \sin \theta+h(\theta$ is the centrifugal angle for the ellipse). $M$ is a point on the oval curve with the angle $\theta$, SMC line is perpendicular to the oval curve on point $M$, and the coordinate of 3 points is $S\left(Z_{S}, R_{S}\right), M\left(Z_{M}, R_{M}\right)$, and $C\left(Z_{C}, R_{C}\right)$, respectively $[17,18]$. The normal equation is

$$
\frac{R_{S}-R_{M}}{a^{2}\left(R_{M}-h\right)}=\frac{Z_{S}-Z_{M}}{b^{2} Z_{M}} .
$$

The effective diameter $D$, the smallest wheel diameter $D_{0}$, and the width of the torus $W$ are known. Thus, the effective total area of torque converter is

$$
F_{D}=\frac{\pi D^{2}}{4} .
$$

The current cross section area $F_{M}$ is as follows:

$$
F_{M}=k F_{D},
$$

where $k$ is the empirical coefficient between 0.166 and 0.27 and statistics suggest that the best value is 0.23 [19]. The intermediate streamlines divide current cross section made up of the outer circle and the inner circle equally. Thus, point $M$ would divide $F_{M}$ equally [20], and the equations could be expressed as

$$
\begin{aligned}
& \frac{\pi}{\cos (\pi / 2-\theta)}\left(R_{S}^{2}-R_{M}^{2}\right)=\frac{F_{M}}{2}, \\
& \frac{\pi}{\cos (\pi / 2-\theta)}\left(R_{M}^{2}-R_{C}^{2}\right)=\frac{F_{M}}{2} .
\end{aligned}
$$

At the moment, $S$ and $C$ are a point on the outer and inner circles, respectively, with a certain step $\Delta \theta$. When $\theta$ starts from 0 to $2 \pi$, the tracks of $S$ and point $C$ would form the outer and inner circles, respectively, $\Delta \theta$ becomes smaller, and the design accuracy increases. When $\theta$ starts from 0 and $\pi$, the point $R_{S}=h$, the boundary of the outer circle, would reach the maximum; the absolute value of $Z_{S}$ is equal to the half of width $W$. Then, the expression of $a$ is as follows:

$$
a=\frac{W-F_{m} / 2 \pi h}{2} .
$$

When $\theta$ reaches $\pi / 2, R_{S}=D / 2$; then the expression of $R_{H}$ is as follows:

$$
R_{H}=\sqrt{\left(\frac{D}{2}\right)^{2}-\frac{F_{m}}{2 \pi}} .
$$

When $\theta$ reaches $3 \pi / 2, R_{S}=D_{0} / 2$, and the expression of $R_{L}$ is

$$
R_{L}=\sqrt{\left(\frac{D_{0}}{2}\right)^{2}+\frac{F_{m}}{2 \pi}} .
$$

As shown in Figure 1, the length of axial $b$ is

$$
b=\frac{R_{H}-R_{L}}{2} .
$$

The center coordinate of the oval is $(0, h)$, and the expression of $h$ is

$$
h=\frac{R_{H}+R_{L}}{2} .
$$

TABLE 1: Parameters of YH245 of different flatness ratios.

\begin{tabular}{lcccc}
\hline & \multicolumn{4}{c}{ Type } \\
& $\mathrm{a}$ & $\mathrm{b}$ & $\mathrm{c}$ & $\mathrm{d}$ \\
\hline Width $(B / \mathrm{mm})$ & 69 & 63.5 & 58 & 52.5 \\
Traditional flatness ratio $(e)$ & 0.28 & 0.26 & 0.24 & 0.21 \\
New flatness ratio (FR) & 0.9 & 0.8 & 0.7 & 0.6 \\
\hline
\end{tabular}

2.2. Parameters of the Outer and Inner Circles. A series of coordinates of points $S$ and $C$, which form the outer and inner circles, respectively, could be obtained from the parameters of the intermediate flow line; the coordinate's expression $R_{S}$ of the outer circle is

$$
R_{S}=\sqrt{R_{M}^{2} \pm \frac{F_{m}}{2 \pi \sqrt{1+b^{4} Z_{M}^{2} / a^{4}\left(R_{M}-h\right)^{2}}}} .
$$

According to (2), the expression of $Z_{S}$ is as follows:

$$
Z_{S}=Z_{M}+\frac{\left(R_{S}-R_{M}\right) b^{2} Z_{M}}{a^{2}\left(R_{M}-h\right)} .
$$

When $\theta$ exceeds $\pi$, values of this formula would become negative; otherwise, they would become positive. Similarly, the coordinate's expression $R_{C}$ of the inner circle can be obtained as follows:

$$
R_{C}=\sqrt{R_{M}^{2} \pm \frac{F_{m}}{2 \pi \sqrt{1+b^{4} Z_{M}^{2} / a^{4}\left(R_{M}-h\right)^{2}}}} .
$$

When $\theta$ exceeds $\pi$, values of this formula would become positive; otherwise, they would become negative. The expression of $Z_{C}$ is follows:

$$
Z_{C}=Z_{M}-\frac{\left(R_{M}-R_{C}\right) b^{2} Z_{M}}{a^{2}\left(R_{M}-h\right)} .
$$

The torus design of elliptic type method could ensure that the design flow line and element line are perpendicular to each other while avoiding a crowd of corrections, the flow line designed is smooth, and the curvature is continuous. Parameters of YH245 of different flatness ratios are displayed in Table 1, and 4 tori of different flatness ratio are shown in Figure 2.

\section{Numerical Calculation Methods and Experiment of Flat TC}

3.1. Governing Equation. We utilized large eddy simulation as it is very efficient at dealing with turbulence flow. In LES, an implication theory of self-similarity states that large flow eddies depend on geometry, whereas smaller ones are more universal. This feature allows explicitly solving for large eddies during calculation and implicitly accounting for small eddies by using a subgrid-scale model [21]. The governing equations used in the LES model were obtained by filtering 


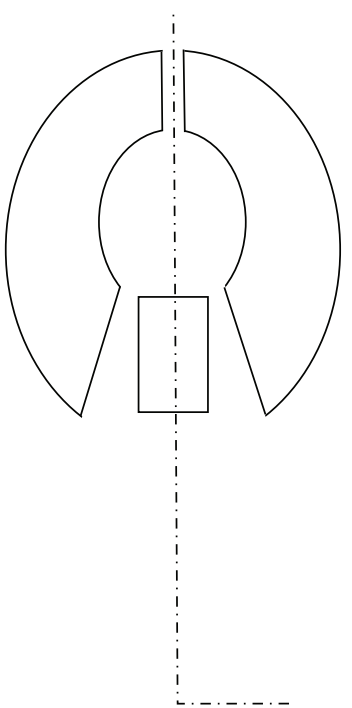

(a) 0.9

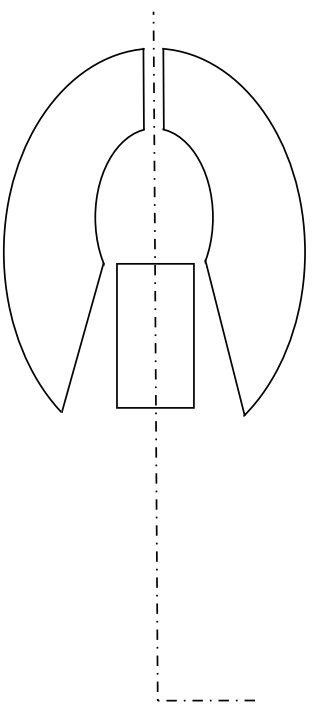

(b) 0.8

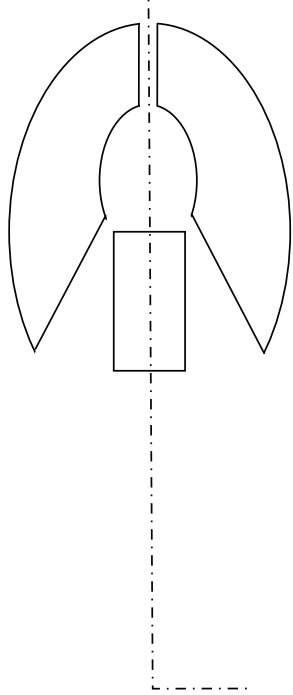

(c) 0.7

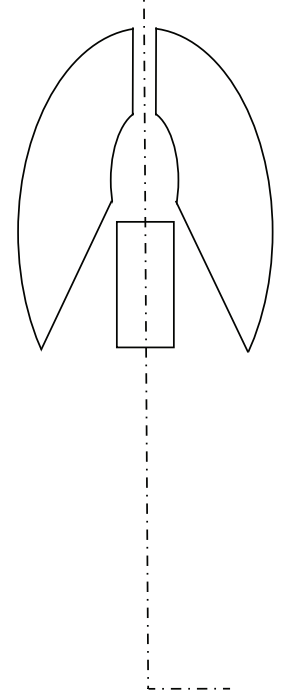

(d) 0.6

FIgURE 2: Tori of different flatness ratios.

the time-dependent incompressible Navier-Stokes equations as follows:

$$
\begin{gathered}
\frac{\partial\left(\rho \overline{u_{i}}\right)}{\partial x_{i}}=0 \\
\frac{\partial\left(\rho \overline{u_{i}}\right)}{\partial t}+\frac{\partial\left(\rho \overline{u_{i} u_{j}}\right)}{\partial x_{j}}=-\frac{\partial \bar{p}}{\partial x_{i}}+\frac{\partial}{\partial x_{j}}\left(\mu \frac{\partial \overline{u_{i}}}{\partial x_{j}}\right)+\frac{\partial \tau_{i j}}{\partial x_{j}}
\end{gathered}
$$

where $\overline{u_{j}}, \bar{p}$, and $\rho$ represent the filtered mean velocity, filtered pressure, and density, respectively; the subgrid-scale Reynolds stress is

$$
\tau_{i j}=-2 v_{T} \overline{S_{i j}}+\frac{1}{3} \tau_{k k} \delta_{i j}
$$

where the rate of strain tensor $\overline{S_{i j}}$ is

$$
\overline{S_{i j}}=\frac{1}{2} \cdot\left[\left(\frac{\partial \overline{u_{i}}}{\partial x_{j}}\right)+\left(\frac{\partial \overline{u_{j}}}{\partial x_{i}}\right)\right] .
$$

$u_{t}$ is the subgrid-scale turbulent viscosity and was modeled by employing the Smagorinsky model as follows:

$$
\mu_{t}=\rho L_{s} \sqrt{2 \overline{S_{i j} S_{i j}}}
$$

$L_{s}$ is the mixing length for subgrid-scale and is defined as

$$
L_{s}=\min \left(k \delta, C_{s} V^{1 / 3}\right),
$$

where $k$ is the von Karman constant and is considered as being $0.41 ; C_{s}$ is the Smagorinsky constant and is considered as being 0.1 .

3.2. Computational Setup. ANSYS FLUENT software was employed to solve the incompressible Navier-Stokes equations without heat transfer [22, 23]. During calculation,
TABLE 2: CFD model description.

\begin{tabular}{lc}
\hline Analysis type & Transient state \\
Turbulence model & LES \\
Pressure-velocity coupling & SIMPLE \\
Transient formulation & Second-order implicit \\
Pump status & Fixed at $2000 \mathrm{rpm}$ \\
Turbine status & Varied from 0 rpm to $2000 \mathrm{rpm}$ \\
Stator status & Stationary \\
\hline
\end{tabular}

the pump and the turbine rotate along the gird interface according to a certain time step size $(0.0005 \mathrm{~s})$. The solution was assumed to have converged when the rate of change in the mass flow rate in two consecutive circulatory iterations and all normalized residuals in mass and momentum conservation equations were less than $10^{-4}$ for the differences of results in these criteria not obvious [24]; the total time step is 200 . The rotational speed of pump is the same with experiment. Mesh interfaces were arranged on the intersecting surfaces of different impellers. The properties of CFD model are summarized in Table 2.

The complex geometry of hydrodynamic elements was accurately represented and the computational meshes were appropriately distributed with ANSYS ICEM computational fluid dynamics (CFD) software to generate the structured hexahedral grid of the entire TC. Mesh-point clustering was performed near the boundary layer, and cell deformity was checked with the software. Figure 3 describes the generation process of the structured grid of entire passage. A mesh sensitivity test in the LES model was performed to minimize the effect of number error. The number of grids is 325,178 , $715,445,1,378,562$, and $1,765,797$, respectively.

3.3. Experiment on Flat TC. The experiment on performance characteristics of TC was carried out through the test 


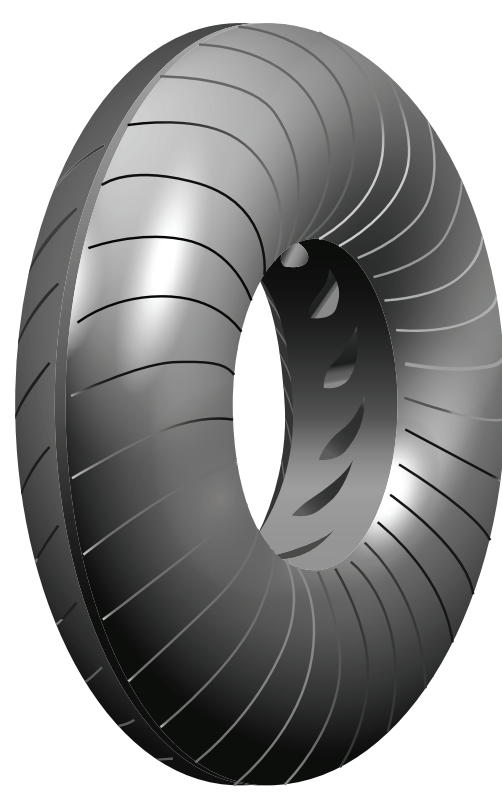

(a)

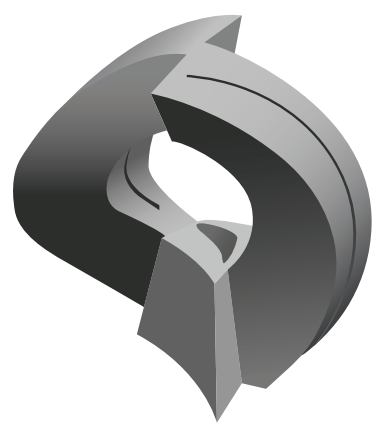

(b)

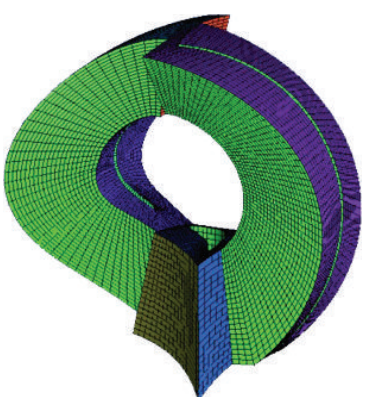

(c)

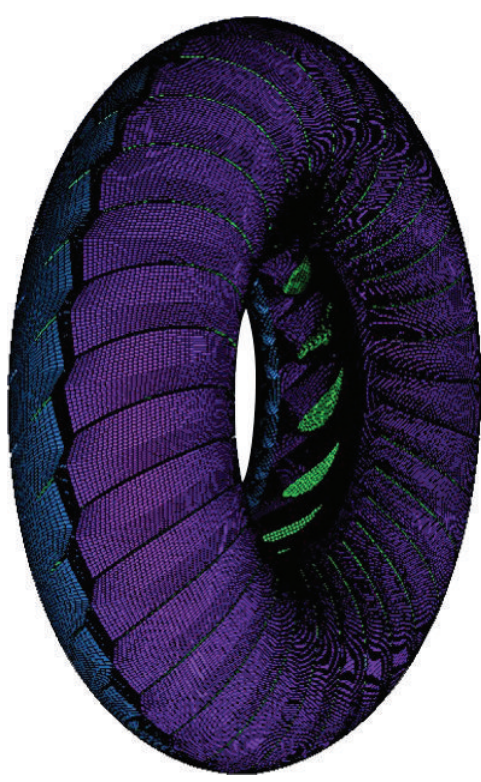

(d)

FIGURE 3: Generation process of the structured grid of entire passage: (a) entire passage, (b) single passage, (c) single structured grid model, and $(\mathrm{d})$ entire passage of the structured hexahedral.

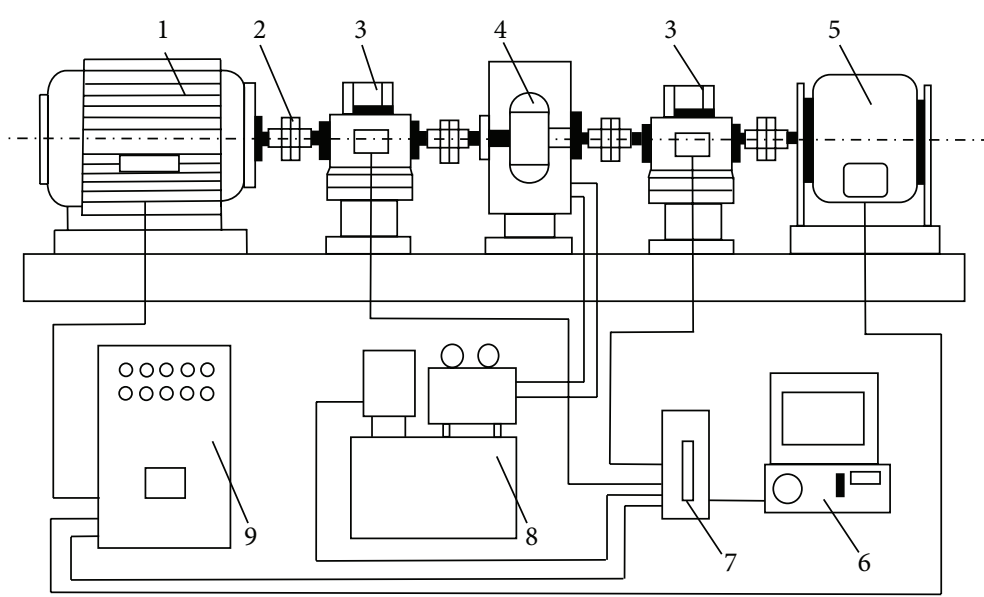

FIGURE 4: Schematic diagram of test platform for hydraulic torque converter: 1: drive; 2: coupling; 3: sensor; 4: torque converter; 5: loading device; 6: industrial control computer; 7: signal converting card; 8: hydraulic station; 9: control cabinet.

platform shown in Figure 4 by Zhejiang Torque Converter Technology Co., Ltd., in November 2011. The performance of a TC is defined by several operating parameters that are described as follows.

SR (Speed Ratio). Consider

$$
\mathrm{SR}=\frac{N_{\text {turbine }}}{N_{\text {pump }}} .
$$

TR (Torque Ratio between the Pump and the Turbine). Consider

$$
\mathrm{TR}=\frac{T_{\text {turbine }}}{T_{\text {pump }}} .
$$

CF (Capacity Factor). Consider

$$
\mathrm{CF}=\frac{T_{\text {pump }} \times 10^{6}}{N_{\text {pump }}{ }^{2}} .
$$



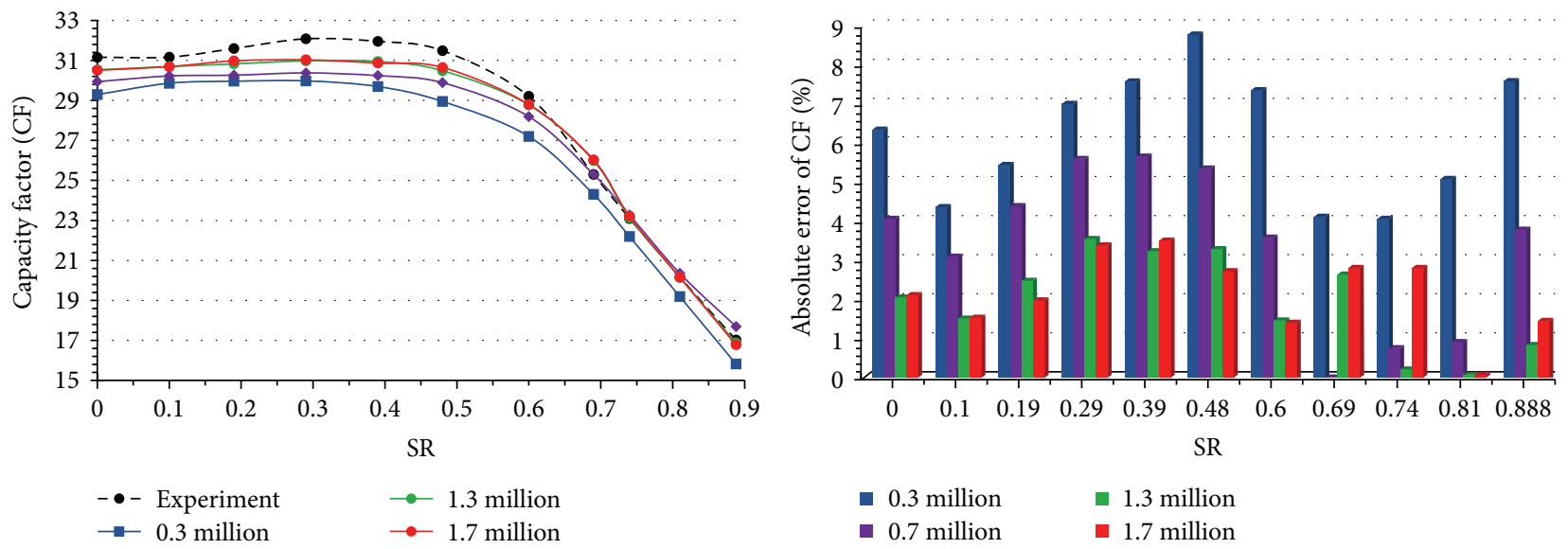

(a) $\mathrm{CF}$ and $\mathrm{CF}$ absolute error
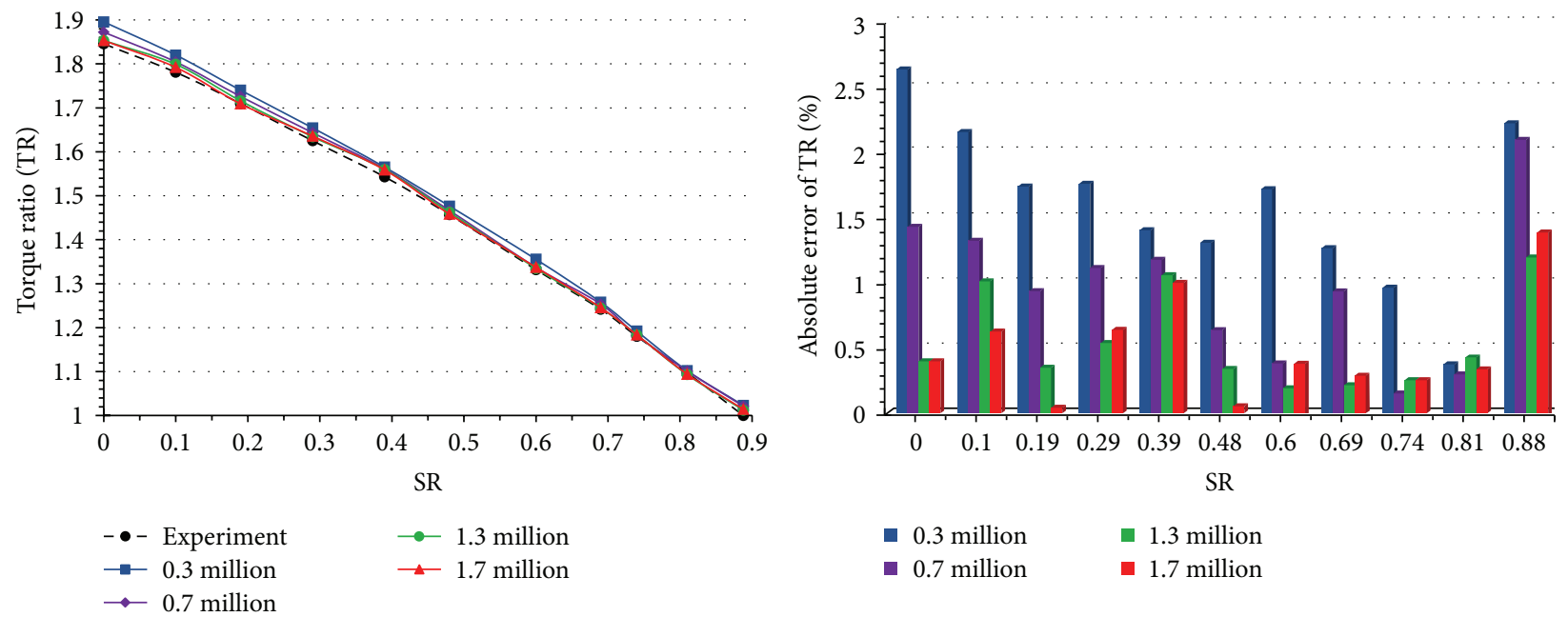

(b) TR and TR absolute error

FIgURE 5: Results of CFD analysis of different mesh densities.

$\eta$ (Efficiency). Consider

$$
\eta=\frac{N_{\text {turbine }} \times T_{\text {turbine }}}{N_{\text {pump }} \times T_{\text {pump }}}=\mathrm{SR} \times \mathrm{TR},
$$

where $N$ is the rotating speed (rpm) and $T$ is the torque of each component. To compare prediction accuracy under different grid densities, the absolute error of prediction is defined as follows:

$$
E_{t}=\frac{\left|I_{\mathrm{TM}}-I_{\mathrm{Exp}}\right|}{I_{\mathrm{Exp}}} \times 100 \%,
$$

where $I$ represents the capacity coefficient, torque ratio, efficiency, and other physical quantities and the subscripts TM and Exp denote the simulation and test data, respectively.

Figure 5 illustrates that the simulated results moved closer to the experimental data with increasing element numbers. Only subtle changes were observed when element numbers increased from 1.3 million to 1.7 million. After an evaluation of computation amount and accuracy, 1.3 million was used in subsequent CFD simulations.

\section{Analysis and Discussion}

4.1. Performance Comparison of Different Flatness Ratio. The measured overall performance of test torque converters is summarized in Figure 6 in terms of the efficiency, capacity factor, and volume flow rate under $S R=0.81$ condition where vehicles are operated most frequently in driving.

Overall efficiency begins to decrease from FR $=0.9$ to $\mathrm{FR}=0.6$ in Figure 6(a). It decreases by no more than 1.0 point from $\mathrm{FR}=0.9$ to $\mathrm{FR}=0.8$ but dramatically decreases from $F R=0.8$ to FR $=0.6$. Figure $6(\mathrm{~b})$ shows the computed torque capacity coefficient and circulatory flow rate. The capacity factor and volume flow rate begin to decrease from $\mathrm{FR}=0.8$ to $\mathrm{FR}=0.6$. The tendency of the torque capacity coincides relatively well with that of the volume flow rate. Figure 6 indicates a significant deterioration in its performance potential along with the narrower profile. 


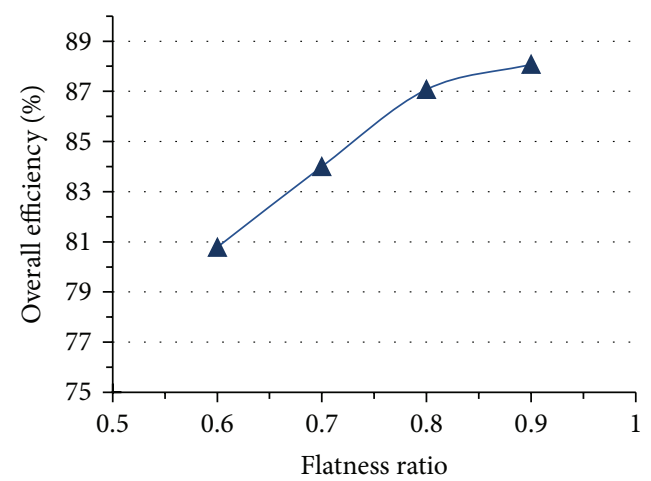

(a) Overall efficiency

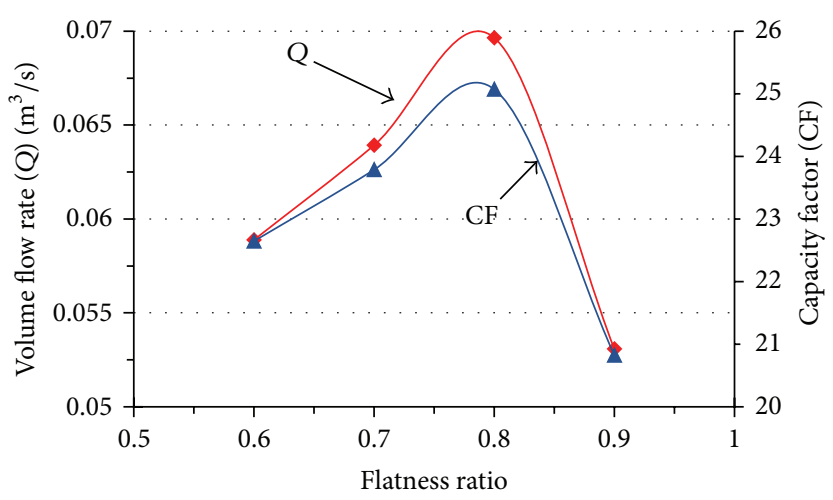

(b) Capacity factor and volume flow rate

FIGURE 6: Overall performance under $\mathrm{SR}=0.81$.

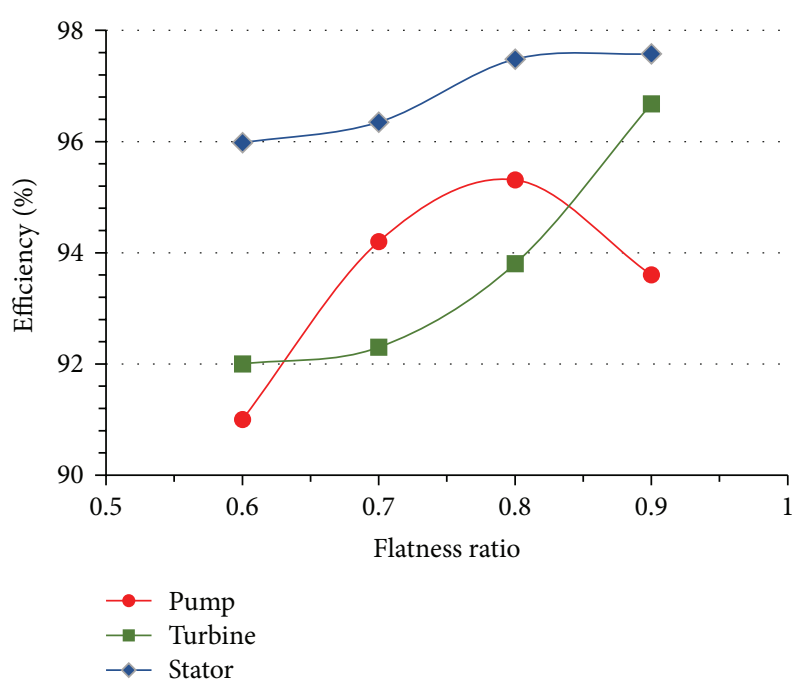

FIGURE 7: Element efficiency under SR $=0.81$.

In order to further investigate the factors influencing performance deterioration, the efficiency of elements will be then discussed.

The individual efficiencies of the pump, turbine, and stator $\left(\eta_{p}, \eta_{T}\right.$, and $\eta_{S}$, resp. $)$ and the overall efficiency $\eta_{\mathrm{TC}}$ are defined by the following expressions:

$$
\begin{aligned}
& \eta_{p}=\frac{\left(p_{p 2}-p_{p 1}\right)}{\rho w_{1}\left(r_{p 2} V_{p 2}-r_{p 1} V_{p 1}\right)}, \\
& \eta_{T}=\frac{\rho w_{2}\left(r_{t 2} V_{t 2}-r_{t 1} V_{t 1}\right)}{\left(p_{t 1}-p_{t 2}\right)}, \\
& \eta_{S}=1-\frac{\left(p_{t 1}-p_{t 2}\right)}{\left(p_{p 2}-p_{p 1}\right)},
\end{aligned}
$$

where subscripts 1 and 2 refer to the inlet and outlet of element, respectively.

Figure 7 displays the efficiency of elements under SR = 0.81, compared with Figure 6(a); the main cause of decline

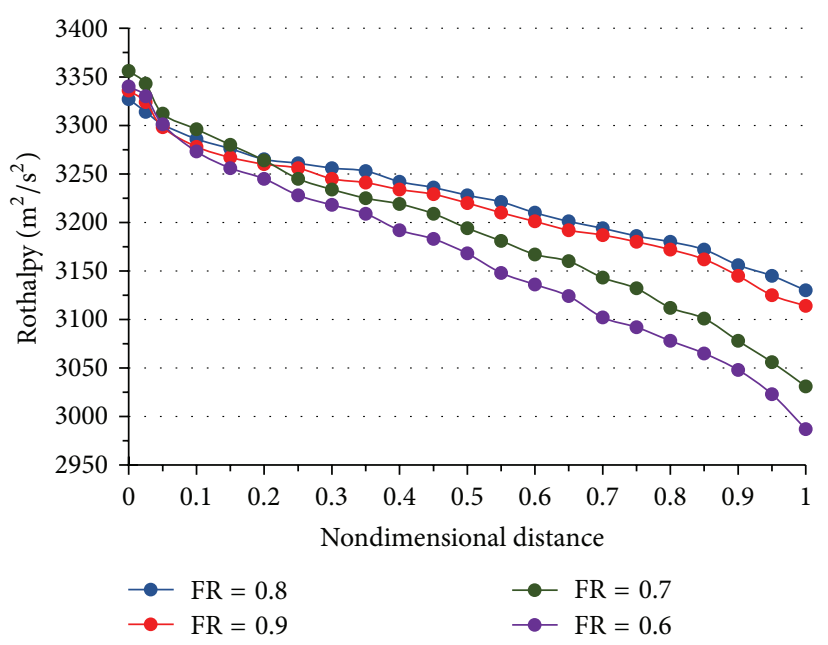

FIGURE 8: Rothalpy change on the pressure surface of pump under $\mathrm{SR}=0.81$

in the overall efficiency is attributed to the pump loss. Here, Rothalpy is introduced to quantitatively evaluate the hydraulic loss. For viscous flow, the difference of parameters between two points on a streamline represents hydraulic loss; Rothalpy is defined as follows:

$$
R=\frac{P}{\rho}+\frac{1}{2} \omega^{2}-\frac{1}{2} \mu^{2},
$$

where $P$ is the pressure, $\rho$ is the density, $\omega$ is the relative velocity, and $\mu$ is the circumferential velocity.

Figure 8 displays the Rothalpy change from the inlet to the outlet on the pressure surface of the pump blades under $\mathrm{SR}=0.81$. The leading edge corresponds to about " $0-0.1$," and the trailing edge corresponds to about " $0.9-1$." The decrease in Rothalpy from FR $=0.9$ to $\mathrm{FR}=0.6$ undoubtedly coincides well with the pump efficiency in Figure 7.

4.2. Performance Comparison of Two Methods. Figure 9(b) shows two kinds of torus; the transitional one is extracted from cross-sectional views of FR $=0.8$ in Figure 9(a). 

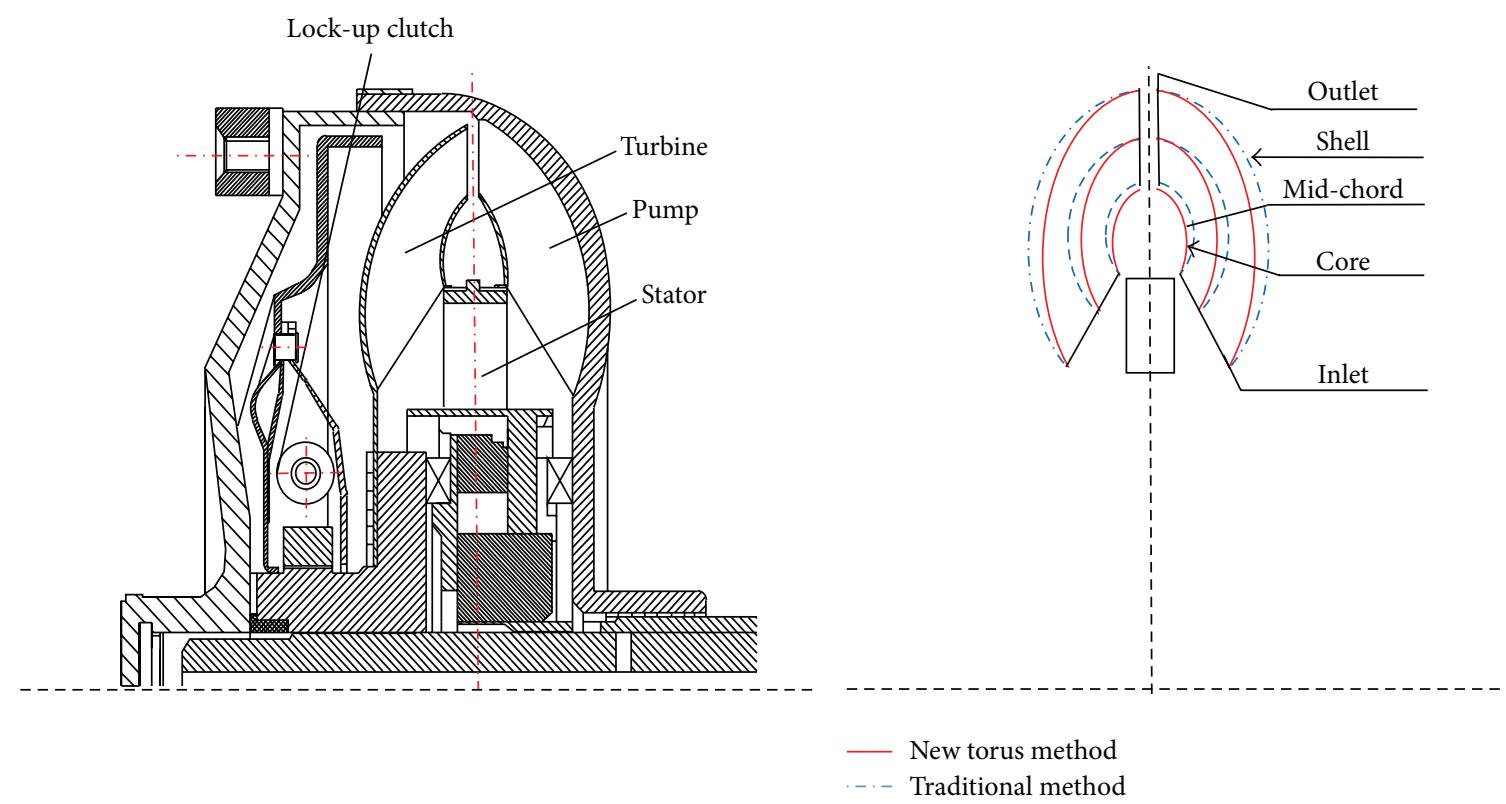

(a) Torus cross-sectional views

(b) Two tori

FIGURE 9: Comparison of torus on different method.

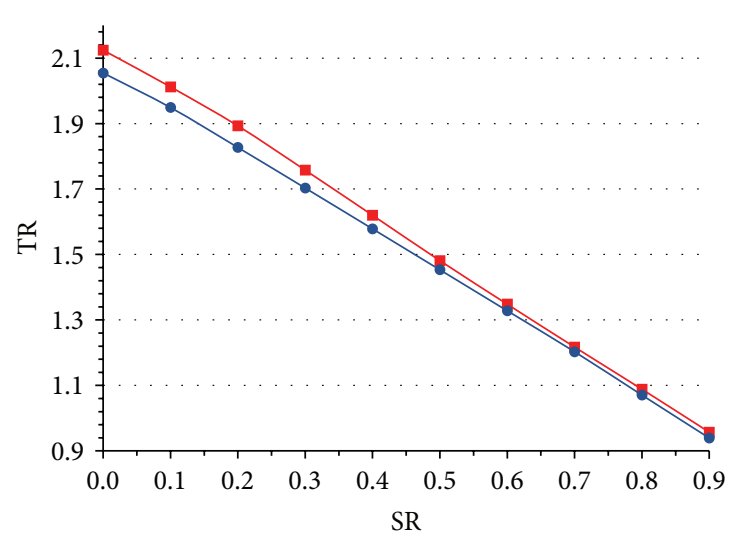

New torus method

Traditional method

(a) Torque ratio

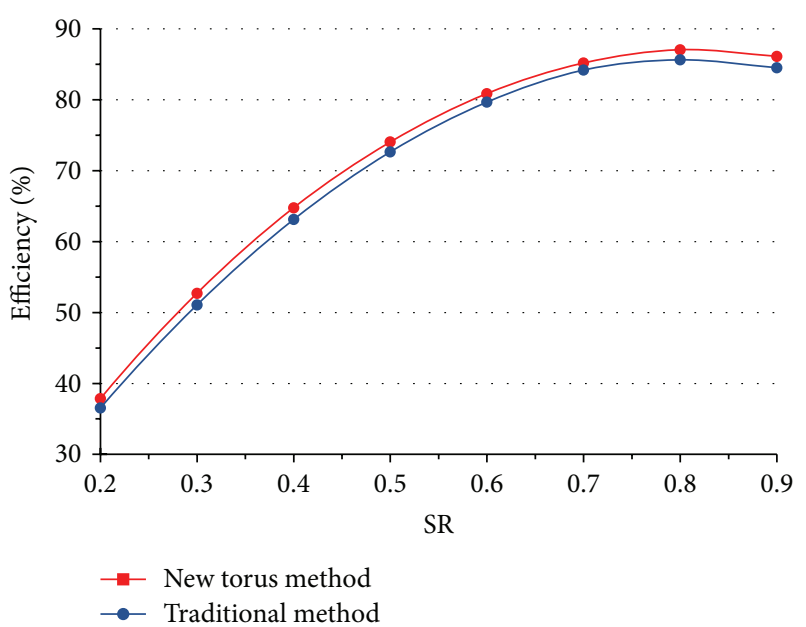

(b) Efficiency

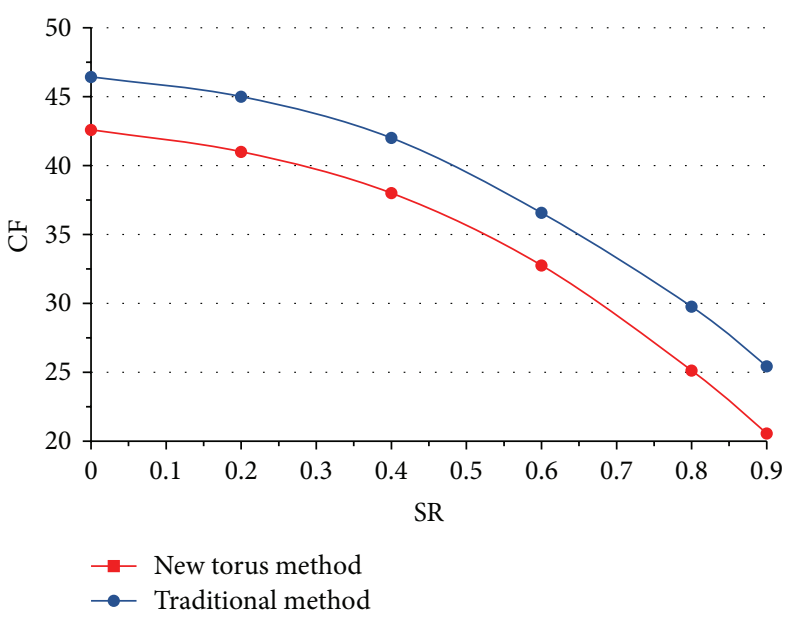

(c) Capacity factor

FIGURE 10: Comparison of performance curves between two methods. 


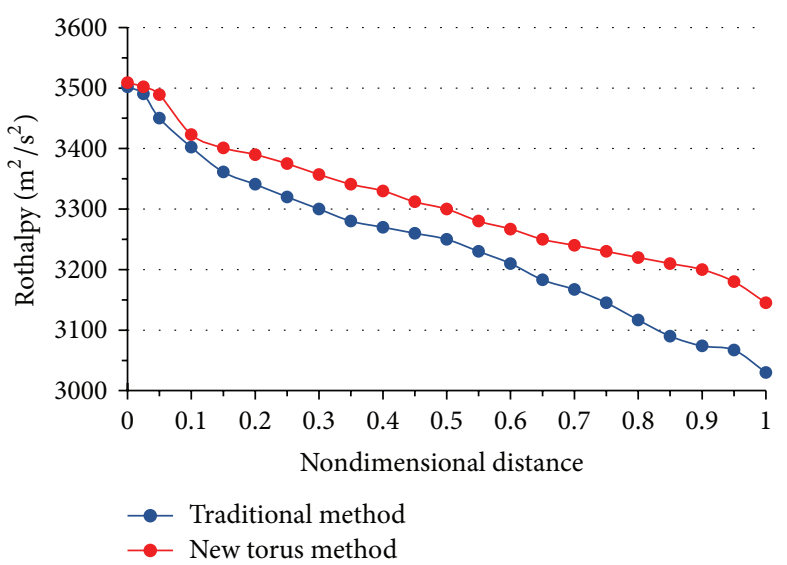

(a) $\mathrm{SR}=0.0$

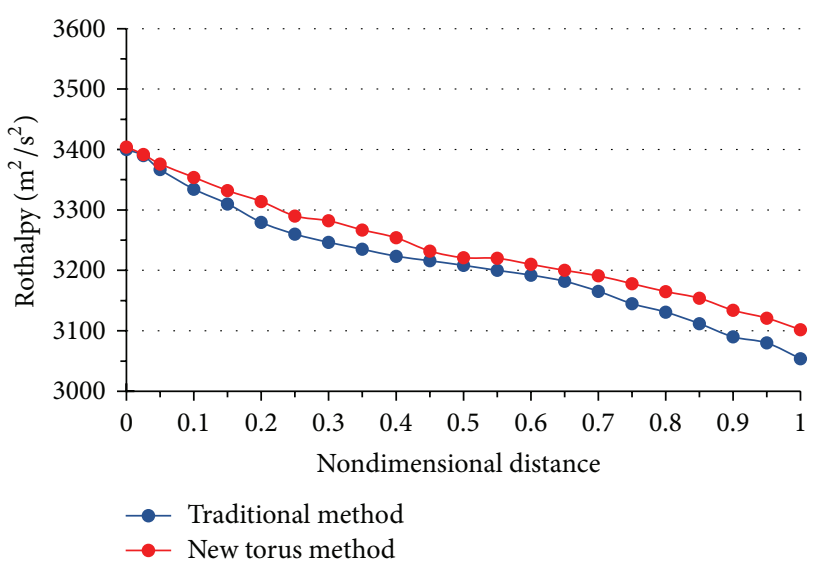

(b) $\mathrm{SR}=0.4$

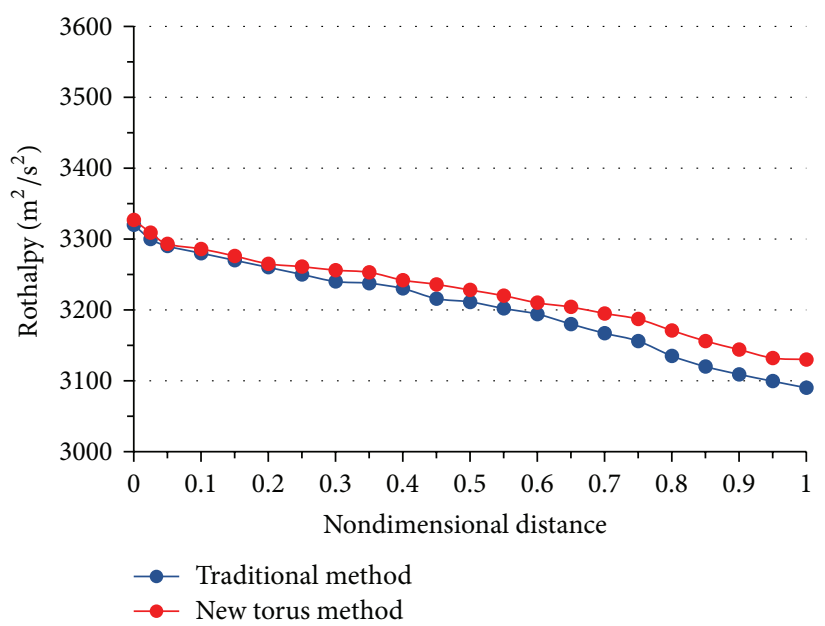

(c) $\mathrm{SR}=0.8$

FIGURE 11: Rothalpy distribution on the pressure surface of pump.

The performance characteristics of the torque converters are investigated by numerical analysis using CFD codes. The results are shown in Figure 10; stall torque ratio is increased from 1.88 to 2.12 ; the highest efficiency on $\mathrm{SR}=0.81$ is improved from $85.6 \%$ to $87.07 \%$, while capacity factor decreases by $12 \%$ compared to the traditional method, which means more energy could be transferred to pump from the engine. In summary, the performance of the flat torque converter designed by adopting the flat torus design method based on the elliptic type is more excellent than that of the traditional design method due to the superior geometry. As the correlation between overall and pump efficiency is quite remarkable in Figure 8, the subsequent discussion will focus on the pump flow and loss.

Figure 11 displays the Rothalpy distribution from inlet to outlet of pump in TC based on two methods. The difference in Rothalpy from inlet to outlet decreases gradually along with SR; compared with the traditional one, Rothalpy distribution in new torus method is gentler and the difference of which is relatively less.

The results illustrate not only the superior geometry of the new method but also the region where large loss occurred with a flatter design. Shock loss owing to the incidence flow angle to the blades changing drastically is attributed to the flow deterioration in the leading edge [25], which accounts for about $20 \%$ of hydraulic loss inside pump; experiment results for the velocity field in a torque converter pump had been carried out by Flack and Brun [26], who had concluded that the strong jet/wake characteristics including backflows and circulatory secondary flows were the main factors contributing to Rothalpy loss in main flow region (0.10.9); complex flow including the free stream flow, blades wake flow, core-suction corner separation, and mixing flow in exit region of pump found by Dong et al. [27] was considered to generate the loss in the trailing edge.

Figure 12 displays the distribution of Rothalpy from the shell to core for the chord plane of pump, where " 0 " refers to the shell surface and " 1 " refers to the core surface.

Type "a" means TC on new method, and the other means traditional one. The Rothalpy difference of any curve on abscissa axes represents the hydraulic loss from shell to core, which would be gradually decreased with the increase of SR. The loss on core surface (the region closing to 1) is mainly focused on the first part of passage, where is located on 


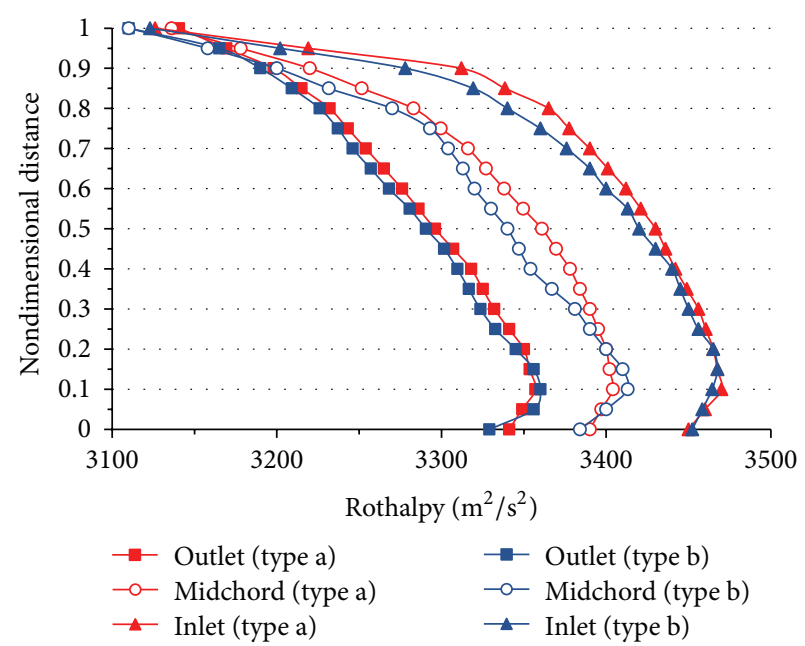

(a) $\mathrm{SR}=0.0$

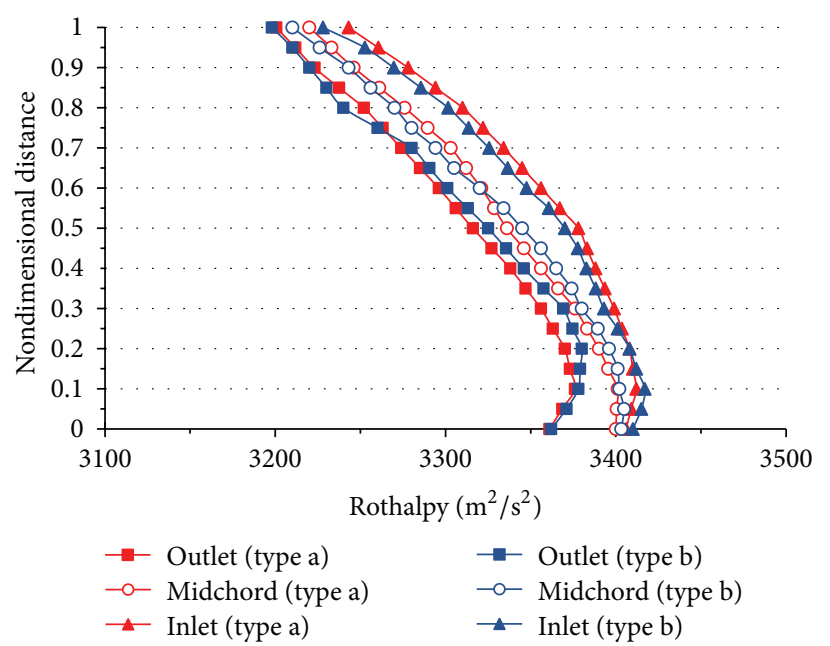

(b) $\mathrm{SR}=0.4$

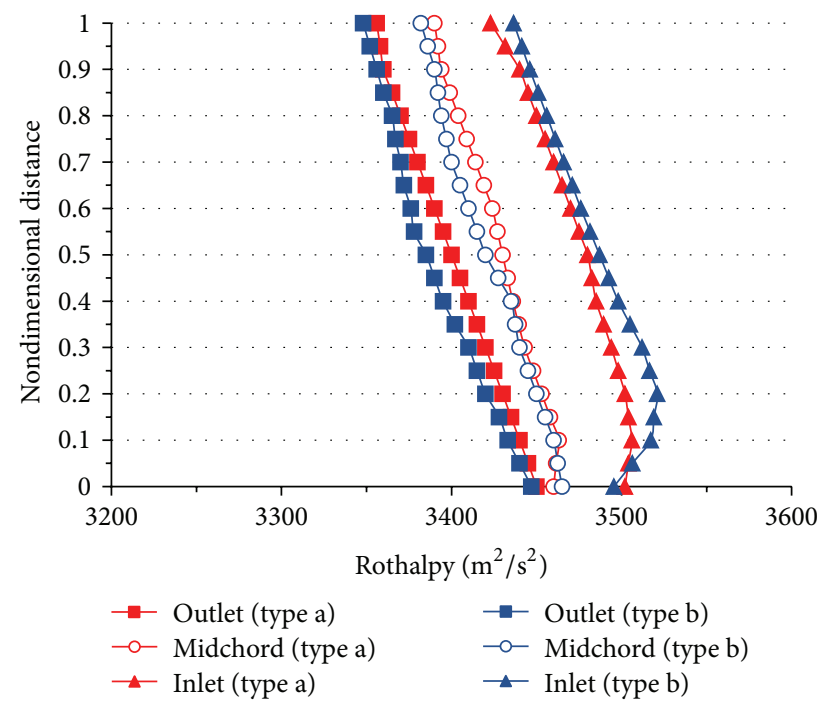

(c) $\mathrm{SR}=0.8$

FIGURE 12: Rothalpy distribution on chord plane of pump.

the region between inlet and mid-chord. By contrast, the loss on the shell surface (the region closing to 0) exhibits an even distribution on inlet, midchord, and outlet. In addition, the Rothaply changes of type a is more smooth and continuous, which suggests the internal flow status of pump on new method is superior to the traditional one.

\section{Influence of Removal of Inner Ring}

In order to further expand the existing space, reduce the torque converter axial size and maintain overall performance of TC; in this paper, three kinds of flatness ratio of TC ( 0.60 , 0.55 , and 0.50 ) without inner ring based on new torus method were designed, as shown in Figure 13.

Figure 14 shows volume flow rate and capacity factor of 4 types of TC on two conditions; comparison on the same flat ratio $(F R=0.60)$ demonstrates the function of inner ring in regulating the volume flow rate and capacity factor.
After removing the inner ring, high-speed working medium flowing from the pump in the radial plane would impact turbine blade more widely, which makes the turbine obtain more power. Furthermore, volume flow rate and capacity factor have lost approximately $15 \%$ of their value when flatness ratio decreased from 0.6 to 0.5 on stall condition $(\mathrm{SR}=0)$, while, on condition of $\mathrm{SR}=0.8$, the difference in 3 types becomes less distinct from each other.

Figure 15 displays the highest efficiency and stall torque ratio on $\mathrm{SR}=0.8$ of the prototype $\mathrm{FR}=0.6$ and 3 kinds of torque converters without the inner ring; a similar pattern in which the highest efficiency and stall torque ratio decrease along with a flatter profile in TC without the inner ring is still valid. At the same time, the comparison between (a) and (b) indicates that the effect of removal of inner ring on improving the performance of existing torque converters is remarkable. The highest efficiency increased by 2.5 percentage points, stall torque ratio is improved from 1.79 to 2.18 , and comparison 


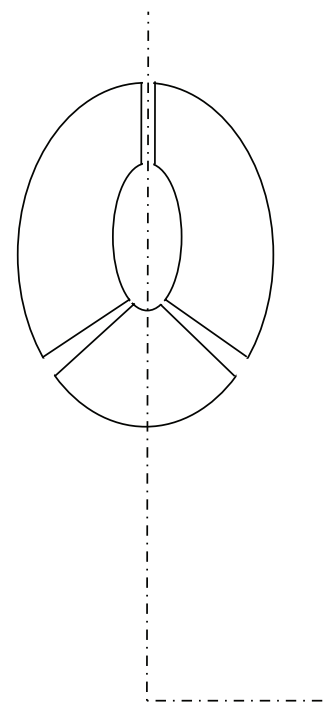

(a) $\mathrm{FR}=0.6$ (inner ring)

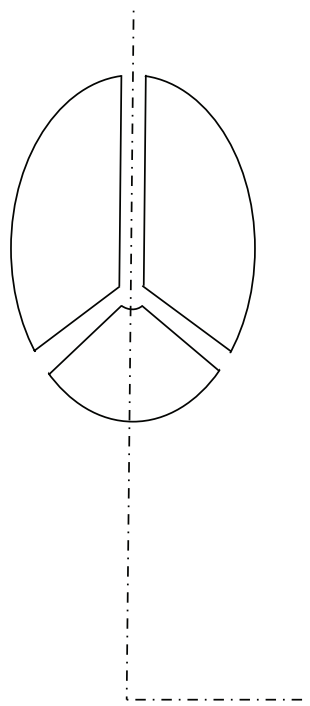

(b) $\mathrm{FR}=0.6$

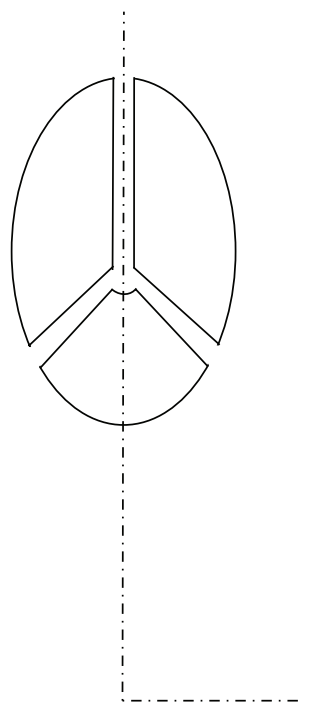

(c) $\mathrm{FR}=0.55$

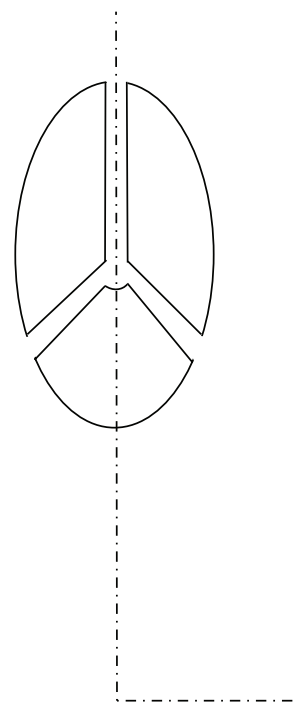

(d) $\mathrm{FR}=0.5$

FIGURE 13: Four types of torus of different flatness ratio.

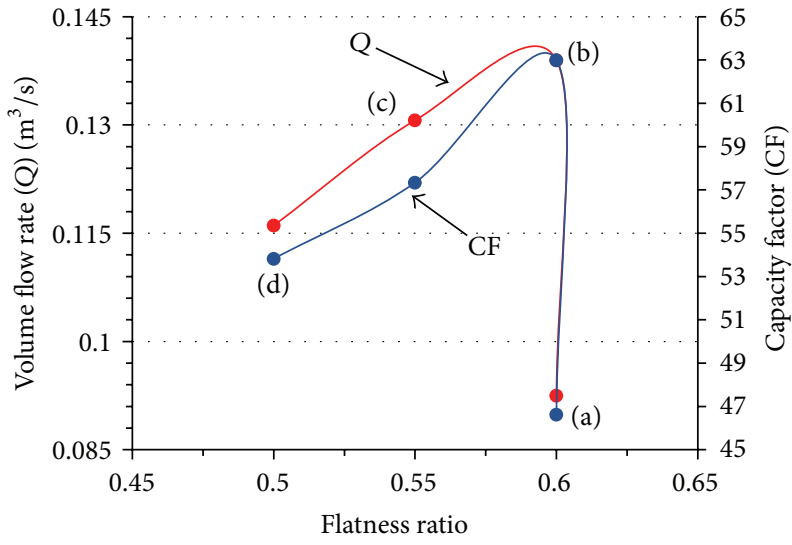

(a) $\mathrm{SR}=0$

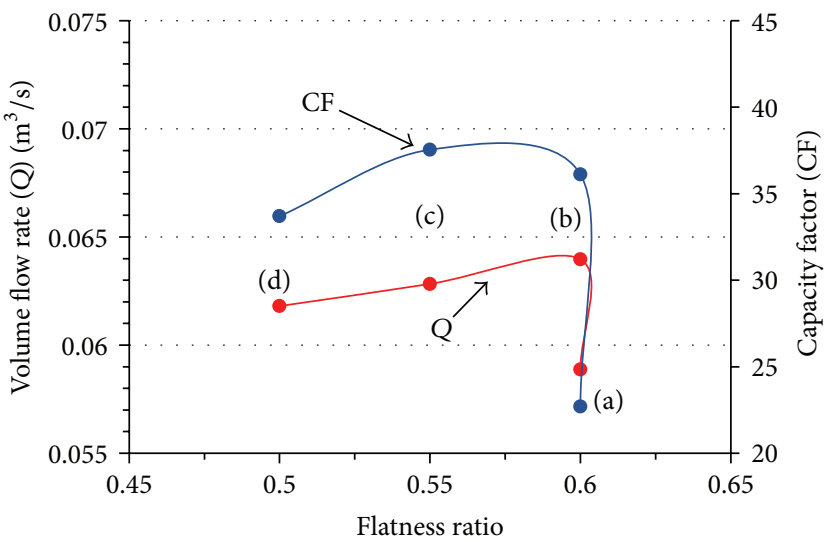

(b) $\mathrm{SR}=0.8$

FIGURE 14: Volume flow rate and capacity factor of 4 types of TC.

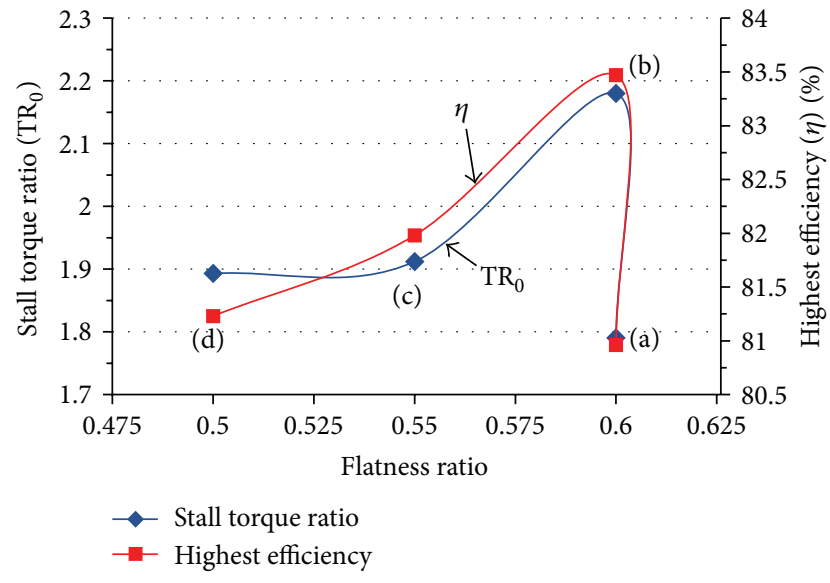

FIGURE 15: Highest efficiency and stall torque ratio of different flatness rate. between (a) and (d) indicates that it is possible to achieve more space and more efficiency and larger stall torque ratio at the same time if the inner ring is removed.

\section{Conclusion}

In this paper, a flat torus of elliptic type method concentrating on the solution to flat TC is put forward to improve the performance. The internal flow characteristics of TC are numerically investigated using CFD codes and resulted in a good agreement with experimental data. Four torque converters of different flatness ratios are designed to judge the superiority of new method; Rothalpy is used to quantitatively evaluate the hydraulic loss and predict the region where large loss occurred with a flatter design. The calculations indicate that the performance of the passenger car flat torque converter deteriorates with the decreasing flatness ratio. 
Furthermore, this paper also proposes a structure of removal of inner ring to improve the performance of TC; the specific conclusions are as follows.

(1) The results indicate that the main cause of this performance degradation can be attributed to deterioration of the velocity fields of the pump.

(2) The performance of the flat torque converter designed by adopting the flat torus design method based on the elliptic shape is more excellent; the internal flow status of pump on new method is superior to the traditional one.

(3) Removal of the inner ring is a feasible way to improve the efficiency while satisfying space requirements.

The stator applied in this paper is straight up and down, called the cylindrical shape. In future studies, other shape-like cones and arc would be checked out to see whether or not the shock loss on leading edge of the pump could be lightened up.

\section{Nomenclature}

CF: Capacity factor

FR: Flatness ratio

$C_{s}$ : Smagorinsky coefficient

$k$ : Turbulent kinetic energy $\left(\mathrm{m}^{2} / \mathrm{s}^{2}\right)$

$N$ : Rotating speed (rpm)

$P: \quad$ Fluid pressure (pa)

$Q:$ Volume flow rate $\left(\mathrm{m}^{3} / \mathrm{s}\right)$

$r$ : Radius (m)

SR: Speed ratio

$T$ : Torque $(n \cdot m)$

TR: Torque ratio between pump and turbine

$u_{i}$ : Time-averaged velocity $(\mathrm{m} / \mathrm{s})$

$X_{i}$ : Coordinate $(\mathrm{m})$.

\section{Greek Letters}

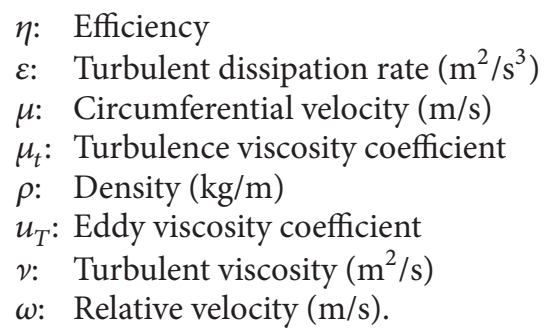

Subscripts

$\begin{array}{ll}P: & \text { Pump } \\ S: & \text { Stator } \\ T: & \text { Turbine } \\ \text { TC: } & \text { Torque converter. }\end{array}$

\section{Conflict of Interests}

The authors declare that there is no conflict of interests regarding the publication of this paper.

\section{Acknowledgment}

This work was supported by the National High-Tech R\&D Program of China (2014AA041502).

\section{References}

[1] T. Ochi, H. Takeuchi, H. Kimura, and K. Watanabe, "Development of a super-flat torque converter for the new toyota FWD 6speed automatic transaxle," SAE Technical Paper 2006-01-0149, 2006.

[2] T. Kietlinski and M. Fingerman, " $248 \mathrm{~mm}$ elliptical torque converter from daimlerchrysler corporation," SAE Technical Paper 2007-01-0241, 2007.

[3] K. Saitou, "Blade structure for torque converter and process of producing the same," U.S. Patent 8,128,370, 2012.

[4] E. Ejiri and M. Kubo, "Performance analysis of automotive torque converter elements," Journal of Fluids Engineering, vol. 121, no. 2, pp. 266-275, 1999.

[5] B. V. Marathe and B. Lakshminarayana, "Experimental investigation of steady and unsteady flow field upstream and downstream of an automotive torque converter pump," International Journal of Rotating Machinery, vol. 5, no. 2, pp. 99-116, 1999.

[6] Y. Dong, V. Korivi, P. Attibele, and Y. Yuan, "Torque converter CFD engineering part I: torque ratio and $\mathrm{K}$ factor improvement through stator modifications," SAE Technical Paper 2002-010883, 2002.

[7] B. S. Kim, S. B. Ha, W. S. Lim, and S. W. Cha, "Performance estimation model of a torque converter part I: correlation between the internal flow field and energy loss coefficient," International Journal of Automotive Technology, vol. 9, no. 2, pp. 141-148, 2008.

[8] J. H. Jung, S. Kang, and N. Hur, "A numerical study of a torque converter with various methods for the accuracy improvement of performance prediction," Progress in Computational Fluid Dynamics, vol. 11, no. 3-4, pp. 261-268, 2011.

[9] C. Liu, A. Untaroiu, H. G. Wood, Q. Yan, and W. Wei, "Parametric analysis and optimization of inlet deflection angle in torque converters," Journal of Fluids Engineering, vol. 137, no. 3, Article ID 031101, 2015.

[10] Y. Dong and B. Lakshminarayana, "Rotating probe measurements of the pump passage flow field in an automotive torque converter," Transactions of the ASME, Journal of Fluids Engineering, vol. 123, no. 1, pp. 81-91, 2001.

[11] J. Schweitzer and J. Gandham, "Computational fluid dynamics in torque converters: validation and application," The International Journal of Rotating Machinery, vol. 9, no. 6, pp. 411-418, 2003.

[12] W. D. Claudel, R. D. Flack, and A. Yermakov, "Average velocity measurements in six automotive torque converters. Part I. Pump measurements," Tribology Transactions, vol. 44, no. 4, pp. 511-522, 2001.

[13] C. Lee, W. Jang, J. M. Lee, and W. S. Lim, “Three dimensional flow field simulation to estimate performance of a torque converter," SAE Technical Paper 2000-01-1146, 2000.

[14] H. S. Su, G. L. Yang, L. Q. Zhang, and W. B. Cao, "Numerical analysis on the external characteristic of torque converter based on UDF," Applied Mechanics and Materials, vol. 318, pp. 44-49, 2013.

[15] R. Flack, "Experimental flow fields in an automotive torque converter-an invited summary and review paper," International Journal of Vehicle Design, vol. 38, no. 2-3, pp. 240-258, 2005. 
[16] E. Ejiri and M. Kubo, "Influence of the flatness ratio of an automotive torque converter on hydrodynamic performance," Journal of Fluids Engineering, vol. 121, no. 3, pp. 614-620, 1999.

[17] S. Liu and L. Quan, "Mathematical model of hydrodynamic torque converter and analytic description of streamline," Chinese Journal of Mechanical Engineering (English Edition), vol. 22, no. 1, pp. 70-77, 2009.

[18] A. Kesy and A. Kadziela, "Application of statistical formulas to hydrodynamic torque converter modelling," Archives of Civil \& Mechanical Engineering, vol. 9, no. 4, pp. 33-48, 2009.

[19] V. J. Janacek, "The design of a single-stage three-element torque converter," SAE Technical Paper 610576, 1961.

[20] S. P. Liu and Q. Long, "Minimum curvature design of threeelement centripetal-turbine hydrodynamic torque converters," Journal of China Ordnance, vol. 5, no. 2, pp. 119-125, 2009.

[21] E. Lenormand, P. Sagaut, L. T. Phuoc, and P. Comte, "Subgridscale models for large-eddy simulations of compressible wall bounded flows," AIAA Journal, vol. 38, no. 8, pp. 1340-1350, 2000.

[22] Y. Lei, C. Wang, Z. Liu, and X. Li, "Analysis of the full flow field of Torque Converter," Advanced Materials Research, vol. 468471, pp. 674-677, 2012.

[23] W. Wei and Q. D. Yan, "Study on hydrodynamic torque converter parameter integrated optimization design system based on tri-dimensional flow field theory," SAE Technical Paper 2008-01-1525, 2008

[24] G. Bois, A. C. Bayeul, C. Leclerc, B. Meredith, and Y. Lebouvier, "Numerical torque converter performance predictions: validation and application to a rapid one dimensional approach," in Proceedings of the ASME Fluids Engineering Division Summer Meeting, Paper no. FEDSM2005-77039, pp. 1095-1102, ASME, Houston, Tex, USA, June 2005.

[25] S. O. Kraus, R. Flack, A. Habsieger, G. T. Gillies, and K. Dullenkopf, "Periodic velocity measurements in a wide and large radius ratio automotive torque converter at the pump/turbine interface," Journal of Fluids Engineering, vol. 127, no. 2, pp. 308316, 2005.

[26] R. Flack and K. Brun, "Fundamental analysis of the secondary flows and jet-wake in a torque converter pump-part I: model and flow in a rotating passage," Journal of Fluids Engineering, vol. 127, no. 1, pp. 66-74, 2005.

[27] Y. Dong, B. Lakshminarayana, and D. Maddock, "Steady and unsteady flow field at pump and turbine exits of a torque converter," Journal of Fluids Engineering, vol. 120, no. 3, pp. 538$548,1998$. 


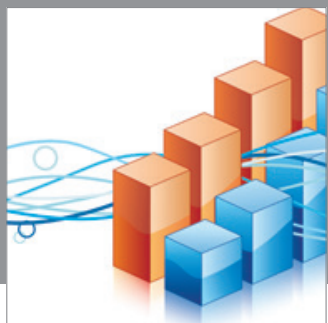

Advances in

Operations Research

mansans

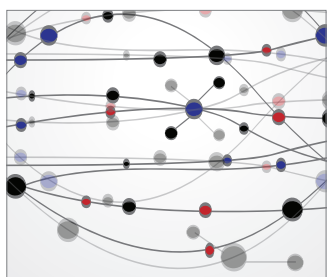

The Scientific World Journal
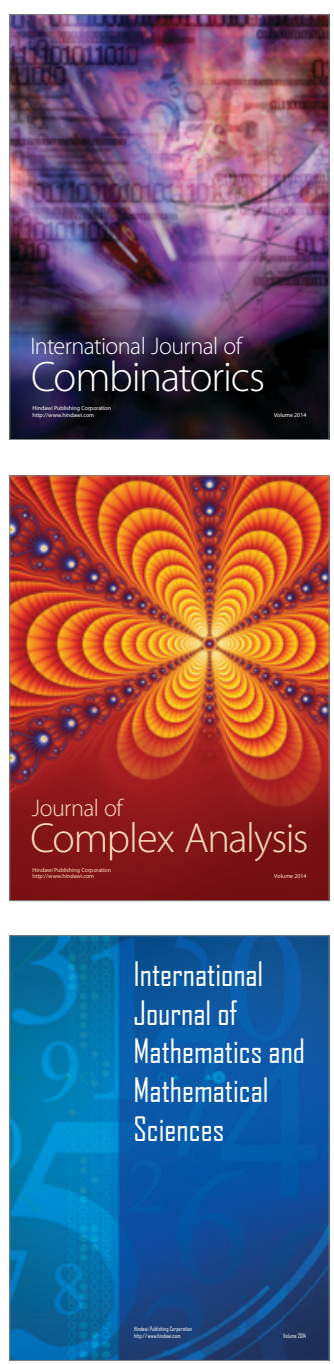
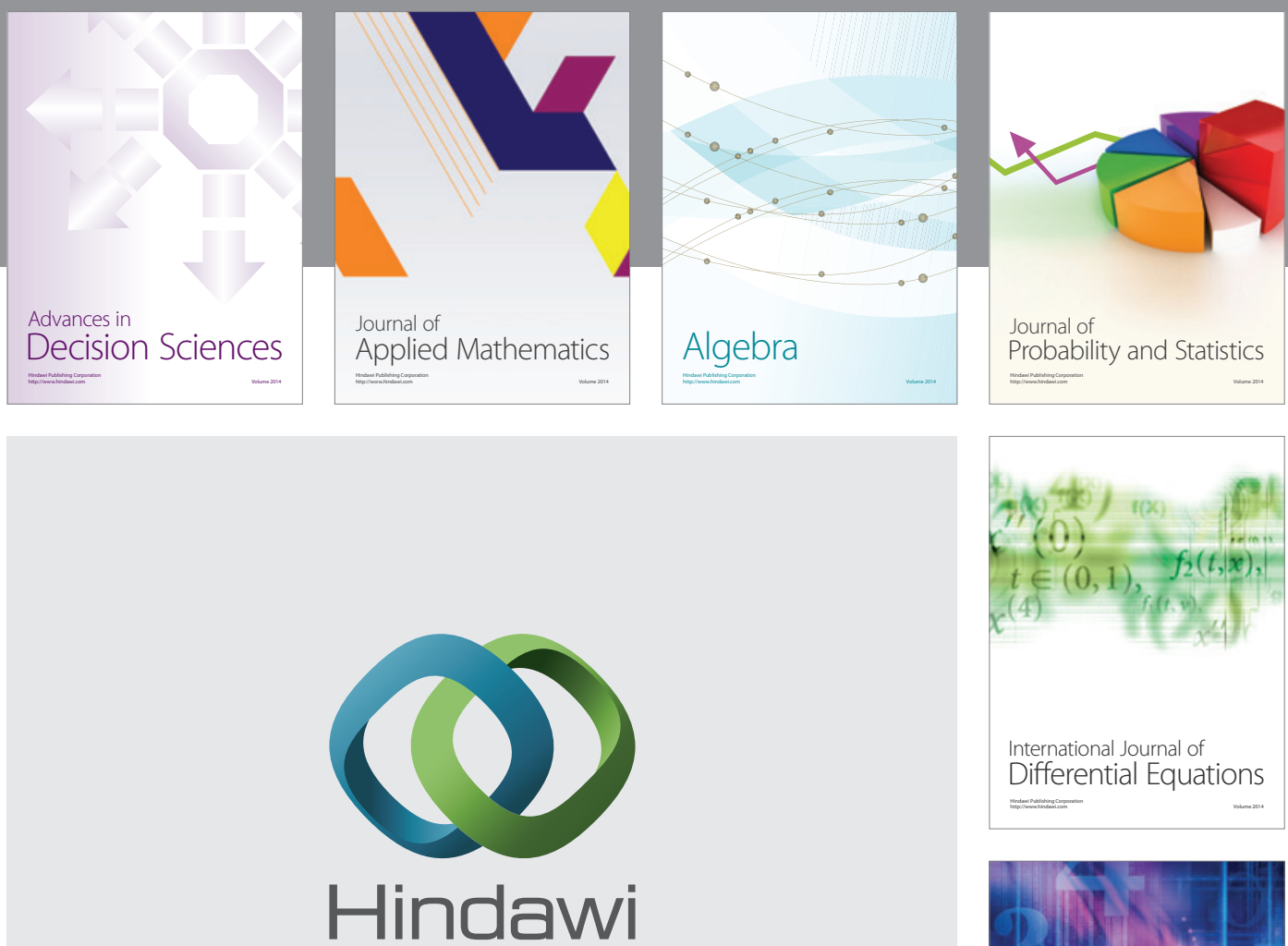

Submit your manuscripts at http://www.hindawi.com
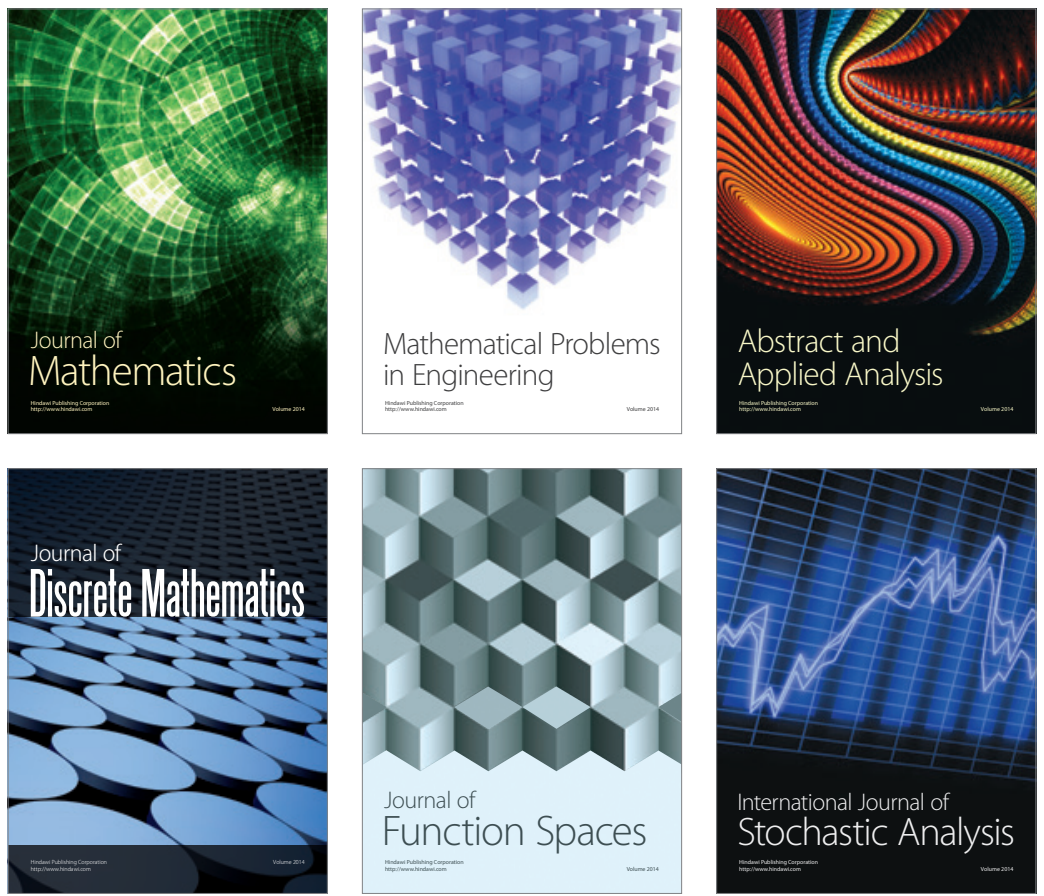

Journal of

Function Spaces

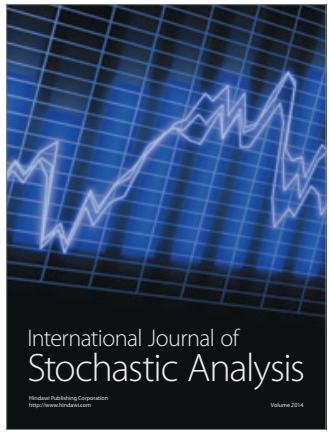

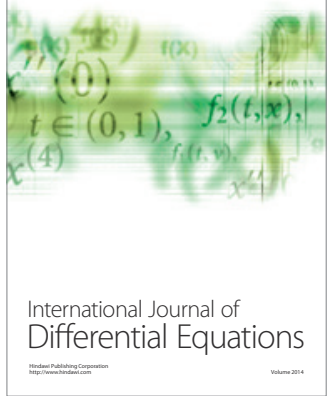
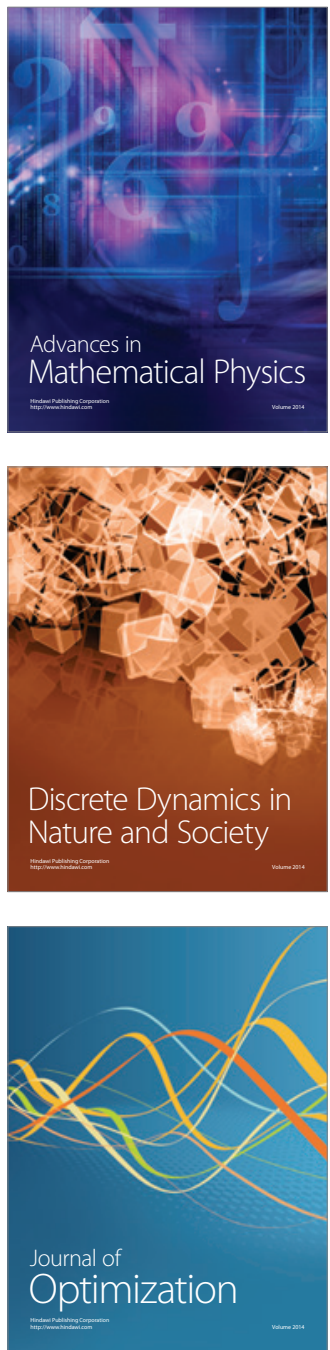